ISSN 1112-9867

Available online at

http://www.jfas.info

\title{
WAVELET SERIES APPROXIMATION USING WAVELET FUNCTION WITH COMPACTLY SUPPORT
}

\author{
E. Tamimi \\ Department of Mathematics, Velayat University, P.O.Box 9911131311, Iranshahr-Iran
}

Published online: 05 June 2016

\begin{abstract}
The Wavelets generated by Scaling Function with Compactly Support are useful in various applications especially for reconstruction of functions. Generally, the computational process will be faster if Scaling Function support descends, so computational errors are summarized from one level to another level. In this article, the convergences of wavelet series are evaluated under certain conditions by applying wavelets with Compactly Support. In fact, an approximation with such wavelets is obtained for wavelet series. This approximation is used in several applications such as compression, decomposition and reconstruction of functions.
\end{abstract}

Keywords: Scaling function; wavelet function; periodic multiresolution analysis (PMRA); wavelet series; compactly support.

Author Correspondence, e-mail: tamimi_ebrahim@yahoo.com

doi: http://dx.doi.org/10.4314/jfas.8vi2s.9

\section{INTRODUCTION}

In general, the Wavelets generated by Scaling Function with Compactly Support are applicable tool to approximate smooth Functions. Also, the compactly supported wavelets have important applications in sobolev spaces [3]. In this regard, M. Skopina in [5] showed that scaling functions with Compactly Support have an important role in convergence and approximation of Wavelet Series. It is well-known that the convergence of Wavelet Fourier Series using terms such as 
decay, Scaling Function convergence and wavelet functions of multiresolution analysis (MRA) have been investigated in some spaces (see [4,5]). Moreover, I. Daubechies in [2] has examined the orthonormal basis of wavelets with compactly support.

In this article, we apply wavelet function (mother wavelet) with Compactly Support instead of scaling function and the investigated studies in the literature are continued with mother wavelet functions. In fact, it is shown that the wavelet (mother wavelet) is applicable tools for approximating Wavelet Fourier Series. Obviously, this type of wavelets play a major role in the development of an alternative algorithm for reconstruction of wavelet-extension based functions.

\section{Prerequisites and definitions}

Suppose that $\mathbb{T}$ is unit circle and $\varphi$ is a scaling function of $L_{2}(\mathbb{R})$ with MRA and for this function, a wavelet function $\psi \in \boldsymbol{L}_{2}(\mathbb{R})$ is exist so that the set $\left\{2^{\mathrm{j} / 2} \psi\left(2^{j} .+n\right)\right\}$ established (for integer values of $\mathrm{n}, \mathrm{j}$ ) an orthogonal bases in $\boldsymbol{L}_{2}(\mathbb{R})$ (see [1]).

If both functions $\psi$ and $\varphi$ are sufficiently damped, the following expression may be written:

$$
\operatorname{Max}(|\varphi(\mathrm{x})|,|\psi(\mathrm{x})|) \leq \frac{\xi}{1+|x|^{1+\epsilon}}, \epsilon>0
$$

Where above inequality is a kind of boundedness for scaling and wavelet function (see [5]). In expression (1), $\xi$ constant is dependent to multiresolution analysis. So by considering the expression (1), it is clear that the following functions (for $\mathrm{j}=0,1,2, \ldots, n=0,1, \ldots, 2^{j}-1$ )

$$
\Phi_{j, n}(\mathrm{x})=2^{j / 2} \sum_{l \in \mathbb{Z}} \varphi\left(2^{j} \mathrm{x}+2^{j} l+n\right), \quad \Psi_{j, n}(\mathrm{x})=2^{j / 2} \sum_{l \in \mathbb{Z}} \psi\left(2^{j} \mathrm{x}+2^{j} l+n\right)
$$

are located in Hilbert space $\boldsymbol{L}_{2}(\mathbb{T})$, and the $\left\{\Phi_{j, n}\right\}_{n=0}^{2^{j}-1}$ and $\left\{\Psi_{j, n}\right\}_{n=0}^{2^{j}-1}$ (for $\mathrm{j}=0,1,2, \ldots$ ) are Orthonormal. In addition, the finite linear combinations $\Psi_{j, n}$ and $\Phi_{j, n}$ :

$$
V_{j}=\operatorname{span}\left\{\Phi_{j, n}, n=0,1, \ldots, 2^{j}-1\right\}, \quad W_{j}=\operatorname{span}\left\{\Psi_{j, n}, n=0,1, \ldots, 2^{j}-1\right\}
$$

are valid in properties of periodic multiresolution (PMRA) (for $\mathrm{j}=0,1,2, \ldots$ ), so we have;

$$
\boldsymbol{L}_{2}(\mathbb{T})=V_{0} \oplus W_{0} \oplus W_{1} \oplus \ldots
$$

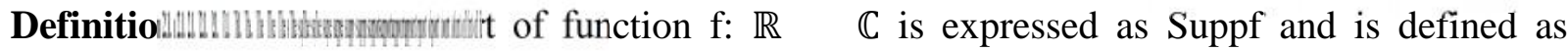
follows:

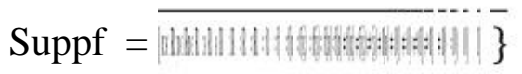

Theorem 2.2. ([6]) Suppose the series are limited as $\sum_{n=0}^{\infty} a_{n}<\infty, \sum_{n=0}^{\infty} b_{m}<\infty$, then: 
A.

$$
\sum_{n=0}^{\infty} a_{n} \sum_{m=0}^{\infty} b_{m}=\sum_{n=0}^{\infty} \sum_{m=0}^{\infty} a_{n-m} b_{m}
$$

B. Suppose double sequence $\left\{a_{i, j}\right\}_{j=1}^{\infty}$ is known so that for $\mathrm{i}=1,2, \ldots, \sum_{j=1}^{\infty}\left|a_{i, j}\right|=b_{i}$ and $\sum_{i=1}^{\infty} b_{i}$ converges. In this case, we have:

Lemma 2.3. ([5]) let be a bounded, decreasing, and integrable function on $[0, \infty)$. Then for all $\mathrm{x}, \mathrm{y} \boldsymbol{\epsilon} \mathbb{R}$, we have:

Where $\mathrm{C}$ is a constant depending only on .

The following lemma already has been proved in [5]. In the next section this lemma is proof by replacing wavelet function rather than scaling function.

Lemma 2.4. ([5]) Let $\mathrm{j}$ be a positive integer, $\mathrm{x}, \mathrm{t} \in\left[0, y_{k}=2^{-j}(t+k), k=0, \ldots, 2^{j}-1\right.$. Then

$$
2^{-j} \sum_{\mathrm{K}=0}^{2^{\mathrm{I}}-1}\left|\sum_{\mathrm{n}=0}^{2^{\mathrm{j}}-1} \Phi_{\mathrm{j}, \mathrm{n}}(\mathrm{x}) \overline{\mathrm{n}}_{\mathrm{n}}\left(\mathrm{y}_{\mathrm{k}}\right)\right| \leq 1
$$

Where is a constant dependent to the PMRA.

\section{THE MAIN RESULTS}

In this section based on Lemma 2.4 and in the form of a proposition, an approximation is obtained for wavelet series by applying wavelet function with compactly support.

Theorem 3.1. Let's assume $\psi$ is a wavelet function with compactly support and is hold in Lemma 2.3, as well as assuming $\mathrm{j}$ is a positive integer and $0 \leq \mathrm{x}, \mathrm{t} \unlhd$. If we substitute $y_{k}=$ $2^{-j}(t+k)$ for $\mathrm{j}=0,1,2, \ldots$ and $k=0, \ldots, 2^{j}-1$ lustrithen we have:

$$
2^{-\mathrm{j}} \sum_{\mathrm{K}=0}^{2^{\mathrm{I}}-1}\left|\sum_{\mathrm{n}=0}^{2^{\mathrm{j}}-1} \Psi_{\mathrm{j}, \mathrm{n}}(\mathrm{x}) \frac{\|}{\mathrm{j}, \mathrm{n}}\left(\mathrm{y}_{\mathrm{k}}\right)\right| \leq C_{\psi} \sum_{k \in \mathbb{Z}} \psi(k)
$$

Where $C_{\psi}$ is constant dependent to the function $\psi$.

Proof . For simplicity, symbols are denoted as followings: 
Since $\psi$ is formed with compactly support, then according to expression (2) (for largely enough values of $\mathrm{j}$ ), we have:

$$
P \leq 2^{j} 2^{-j} \sum_{k=0}^{2^{j}-1}\left|\sum_{n=0}^{2^{j}-1} \sum_{l_{1} \in \mathbb{Z}} \psi\left(2^{j} x+2^{j} l_{1}+n\right) \sum_{l \in \mathbb{Z}} \psi\left(t+k+2^{j} l+n\right)\right|
$$

According to the equation (1) and Lemma 2.3, the convergence of two former sigma in above inequality is evident. Thus, based on the Theorem 2.2 (B), we get:

However, according to the first part of Theorem 2.2 (A), we have:

$$
\begin{aligned}
P \leq & \sum_{k=0}^{2^{j}-1} \sum_{n=0}^{2^{j}-1} \sum_{l \in \mathbb{Z}} \sum_{l_{1} \in \mathbb{Z}}\left|\psi\left(2^{j} \mathrm{x}+2^{j} l_{1}+\mathrm{n}\right)\right| \mid \psi\left(t+k+2^{j}\left(l \pm l_{1}\right)+n \mid\right. \\
& =\sum_{k=0}^{2^{j}-1} \sum_{n=0}^{2^{j}-1} \sum_{l_{1} \in \mathbb{Z}} \sum_{l \in \mathbb{Z}}\left|\psi\left(t+k+2^{j} l+2^{j} l_{1}+n\right)\right|\left|\psi\left(2^{j} \mathrm{x}+2^{j} l_{1}+\mathrm{n}\right)\right| ; \quad m=2^{j} l_{1}+\mathrm{n}
\end{aligned}
$$

However, because $0 \leq \mathrm{x}, \mathrm{t} \leq 1$ and $\mathrm{k}=0,1, \ldots, 2^{j}-1$, then for $l \in \mathbb{Z}$ and $|l| \geq 1$ given that Supp $\psi \subset[-\mathrm{R}, \mathrm{R}]$ in which $0<\mathrm{R}<\infty$, we have:

$$
\operatorname{Supp} \psi\left(2^{j} \mathrm{x}+m\right) \cap \operatorname{Supp} \psi\left(t+k+2^{j} l+m\right)=\emptyset
$$

Now by converting an apparent variable $m$ to $n$ and therefore according to Lemma 2.3 we will obtain: 
So as aforementioned above without loss of generality, we assume $\psi(x)=\psi(|x|)$. Thus we have:

Now we claim that for $k \in \mathbb{Z}$ and $\mathrm{x}, \mathrm{t} \in[0,1]$, the following result is obtained:

For this purpose with $0 \leq \mathrm{x}, \mathrm{t} \leq 1$ and large enough values of $\mathrm{j}$, the following representations are obtained:

$$
\begin{aligned}
& 0 \leq 2^{j} \mathrm{x} \leq 2^{j} \quad 2^{j}-\mathrm{t} \geq 2^{j} \mathrm{x}-\mathrm{t}>-10 \Leftrightarrow \frac{2^{j} \mathrm{x}-\mathrm{t} \pm 2}{4}>-2 \\
& 2^{j}-\mathrm{t} \geq 2^{j} \mathrm{x}-\mathrm{t}>-5 \Leftrightarrow 2^{j} \mathrm{x}-\mathrm{t}+1>-4 \Leftrightarrow \frac{2^{j} \mathrm{x}-\mathrm{t} \pm 1}{4}>-1 \\
& 2^{j} \mathrm{x} \geq t \Leftrightarrow \frac{2^{j} \mathrm{x}-\mathrm{t}}{4}>0, \ldots
\end{aligned}
$$

On the other hand, we have:

$$
+\cdots=G
$$

According to equation (3) to (7) and descending behaviour of $\psi$ and the representation $\psi(x)=\psi(|x|)$, we have:

Therefore, our claim is verified and the proof of the theorem is completed. 


\section{CONCLUSION}

In this article, the compactly support condition is added to such terms as boundedness and dampness of mother wavelet function (wavelet function) of PMRA. It is observed that such wavelets are applicable tool for approximation of wavelet series. Moreover, another result is that with decreasing scaling and wavelet functions support on many topics associated to wavelet, the calculation errors will be reduced and so the reconstruction of functions that are established based on wavelet extensions will be easier and enhanced than before.

\section{REFERENCES}

[1] Daubechies I. Ten lectures on wavelets, CBMS/NSF Regional Conference Series in Applied Mathematics. Society for Industrial and Applied Mathematics (SIAM), 1992, 61, 128-149.

[2] Daubechies I. Orthonormal bases of compactly supported wavelets. Comm Pure Appl. Math. 1988, 41, 909-996.

doi : 10.1002/cpa.3160410705

[3] Isuki M. The compactly supported wavelet characterization of non-homogeneous Herzsobolev spaces with variable exponent. Analysis Mathematica., 2012, 33, 19-40. doi : 10.1007/g10476-012-0102-y.

[4] Skopina M. Multiresolution Analysis of Periodic Functions. EJA., 1997, 3, 203-224.

[5] Skopina M. Wavelet Approximation of Periodic Functions. The Journal of Approx Theory. 2000, 104 (2), 302-329. doi: 10.1006/jath.1999.3434

[6] Rudin W. Principles of Mathmatical Analysis, $3^{\text {rd }}$ ed. McGraw-Hill, New York, 1976, 175178.

\section{How to cite this article:}

Tamimi E. Wavelet series approximation using wavelet function with compactly support. J. Fundam. Appl. Sci., 2016, 8(2S), 132-137. 